\title{
LE SYSTÈME BRÉSILIEN DE PROTECTION ET DE DÉFENSE DU CONSOMMATEUR
}

\section{Étienne Chénier-Laflèche ${ }^{*}$}

\section{Introduction}

La défense du consommateur fait figure de droit constitutionnel au sein du système juridique brésilien. Après plus de 20 ans de dictature et dans un contexte où l'économie nationale s'intègre de plus en plus au sein de l'économie mondiale, il fut reconnu que la protection des droits des consommateurs ne cherche pas qu'à rééquilibrer les relations de consommation mais constitue aussi une garantie d'intégration citoyenne. En effet, considérant que l'immense majorité de la population joue, d'une manière ou d'une autre, un certain rôle au sein des relations de consommation, l'importance que fut portée à la protection du consommateur témoigne de la reconnaissance que ce dernier est d'abord et avant tout, citoyen. Ainsi, de façon à mieux comprendre le fonctionnement du système brésilien de protection du consommateur, il sera tout d'abord abordé le processus historique qui en marqua la formation. Par la suite, une attention particulière sera portée à la formation du Code de protection et de défense du consommateur et aux principaux droits garantis par celui-ci. Enfin, un survol des institutions veillant à l'application du code, desquelles se démarquent le Département de protection et de défense du consommateur du Ministère de la justice et les Procons estataux et municipaux, sera effectué.

\section{Survol historique}

Le développement du mouvement pour la protection et la défense du consommateur au Brésil fut intimement associé à celui de son économie nationale. En effet, si l'élaboration du Code de protection et de défense du consommateur n'est que très récente, il est à noter que le pays ne présenta les caractéristiques propres à une «société de consommation» que tardivement, au cours des années cinquante.

Ainsi, jusqu'au début des années trente, l'économie brésilienne, soutenue par la culture du café à des fins d'exportations, ne permit pas le développement d'un véritable marché interne ce qui eut pour conséquence de limiter les relations de consommation ${ }^{1}$. À cette époque, le Brésil fut, à toute fin pratique, exclu de la révolution industrielle qui avait alors cours en Europe et en Amérique du Nord. En ce qui a trait à la législation en vigueur, malgré l'adoption d'un nouveau Code civil en 1916, les consommateurs ne sont que très indirectement affectés ${ }^{2}$ par sa lente modernisation.

\footnotetext{
Université du Québec à Montréal, Universidade Federal do Rio Grande do Sul.

Marcelo Gomes Sodré, Formá̧äo do Sistema Nacional de Defesa do Consumidor, São Paulo, Editora Revista dos Tribunais, 2007, p. 244.

2 Fundação Procon de São Paulo, Históño - No Brasil, en ligne: http://www.procon.sp.govbr/texto.asp?id=1146 (page consultée le 10 avril 2008).
} 
Toutefois, à compter de l'abolition officielle de l'esclavage en 1888, une société basée sur la consommation commença lentement à voir le jour. Ce phénomène s'appuya sur le processus d'urbanisation qui résulta de l'important mouvement migratoire initié à la faveur de la crise de la main d'œuvre agricole qui suivit le passage de l'esclavagisme au salariat. ${ }^{3}$

Toutefois, ce n'est qu'à compter de la crise économique et de la Seconde Guerre Mondiale que le Brésil eut l'opportunité de développer son industrie de base et de stimuler véritablement son marché interne. Ainsi, au début des années cinquante, la production industrielle en série marque l'entrée du pays dans la société de consommation. Par conséquent, fut développé entre 1930 et 1960, au sein du droit pénal, une série d'instruments visant à protéger les consommateurs. Toutefois, comme le souligne Marcelo Gomes Sodré, le droit pénal, faisant bien plus figure d'exception que de règle, ne permit pas d'atteindre cet objectif. À cet effet, il souligne que « des questions du quotidien comme la responsabilité civile et la protection contractuelle ne furent l'objet d'aucune initiative. $\rangle^{4}$

À l'aube du retour à la démocratie, marqué par l'arrivé au pouvoir de Juscelino Kubitschek en 1956, le Brésil pouvait alors figurer comme véritable société de consommation. De surcroît, le développement de l'industrie automobile et l'augmentation des investissements internationaux qui marquèrent le mandat de Kubitschek contribuèrent à faire du pays un exportateur de produits manufacturés. Malgré tout, la politique interventionniste suivie par le président Kubitschek et le retour d'un régime autoritaire en 1964 n'altérèrent pas l'influence prédominante qu'avait l'État sur la société.

Conformément à l'omniprésence de l'État, l'évolution du droit de la consommation fut largement influencée par le développement du droit administratif. ${ }^{5}$ Ainsi on assista à la formation de plusieurs systèmes de défense du consommateur, entendu que plusieurs ramifications du pouvoir étatique traitèrent des relations avec les consommateurs, notamment au sein de domaines comme la santé et la concurrence. Á cet égard, la fondation de la SUNAB (Bureau national pour la supervision de l'offre) aurait bien pu jouer un rôle central dans la lutte pour la protection et la défense du consommateur, mais, "confondant la défense du consommateur avec le contrôle des prix ${ }^{6}$, l'organisation n'aura finalement traité que de dossiers mineurs (pourboires dans les restaurants, études sur l'évolution des prix, etc.). Le directeur de la SUNAB a par ailleurs confirmé au cours d'une Commission parlementaire d'enquête que l'organisme n'avait pas comme objectif la protection et la défense des consommateurs. ${ }^{7}$ Par ailleurs, la très grande centralisation de la SUNAB ne favorisa pas le développement d'un système national coordonné et harmonisé, une lacune qui se fit sentir lorsque fut créé un véritable système national de protection du

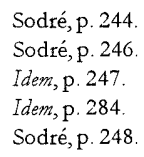


consommateur. Ainsi, en l'absence d'un véritable interlocuteur en matière de défense des consommateurs au niveau fédéral, plusieurs états mirent sur pied leur propre agence de défense du consommateur (mouvement initié par la fondation du Procon de São Paulo en $1976)^{8}$ et de nombreuses organisations civiles virent le jour. Toutefois, si le développement de tels organes constitue un net progrès en la matière, en constituant de véritables interlocuteurs étatiques, celui-ci s'est malheureusement fait au détriment d'un système national harmonisé. ${ }^{9}$ Comme le souligne M. Sodré, cette évolution désarticulée du Système national constitue une occasion ratée considérant que la réelle prise de conscience de l'importance de la défense du consommateur eut lieu simultanément au désengagement de l'État en cours lors des années quatre-vingt-dix. ${ }^{10} \mathrm{Il}$ devenait donc nécessaire de rattraper le temps perdu.

À compter des années quatre-vingt, l'approfondissement de l'intégration de l'économie brésilienne dans le marché global initia plusieurs réajustements du marché interne tels que l'accès facilité au crédit et l'augmentation de la quantité des relations de consommation ${ }^{11}$. Un important rattrapage en matière de protection des consommateurs est alors initié. En effet, tant le développement de l'industrie, l'influence du mouvement international de défense du consommateur, l'organisation de la société civile, la démocratisation du pays, l'arrimage du marché interne au marché international et l'intérêt manifesté par les grandes multinationales contribuèrent à cette évolution. ${ }^{12}$ Par conséquent, tant au niveau législatif qu'au niveau de la maturité de la société civile, le Brésil fit un grand bond en avant.

Ainsi, à la faveur du retour à la démocratie en 1985, plusieurs entités de défense et de protection du consommateur virent le jour. Notons par exemple la création en 1985 du Conseil National de Défense du Consommateur (forum de discussion et d'implémentation des politiques publiques pour la défense du consommateur), la fondation de l'Institut brésilien de défense du consommateur (IDEC) en 1987, l'inclusion dans la nouvelle Constitution de 1988 d'articles traitant spécifiquement de la défense du consommateur, et, l'adoption en 1990 du Code de Protection et de Défense du Consommateur (CDC). Ce dernier prévoit explicitement la création d'un Système National de Défense du Consommateur. Bref, malgré une lente organisation du Système National, nous devons constater le remarquable progrès effectué depuis les vingt dernières années.

\footnotetext{
8 Secretaria de Justiça e da Defesa da Cidadania, PROCON - 25 anos, São Paulo, Governo do Estado de São Paulo, 2001, p. 15 9 Sodré p. 249.

10 Idem, p. 250

11 Rogério Medeiros Garcia de Lima, Aplicasăo do Cóágo de Defesa do Consumidor, São Paulo, Editora Revista dos Tribunais, $2003, \mathrm{p} .32$

12 Sodré, p. 252.
} 


\section{Code de protection et de défense du consommateur}

\section{Formation}

L'adoption de la Constitution fédérale de 1988 initia une nouvelle ère en matière de protection et de défense du consommateur. En effet, au sein de l'article 5 énumérant les droits fondamentaux, on affirme au paragraphe XXXII que «l'État fera la promotion, au moyen d'une loi, de la défense du consommateur ${ }^{1.3}$. Par ailleurs, le paragraphe $\mathrm{V}$ de l'article 170 affirme que la défense du consommateur constitue l'un des piliers de l'ordre économique du pays. ${ }^{14}$ Enfin, il fut spécifié à l'article 48 de l'Acte des dispositions constitutionnelles transitoires que «le Congrès National, dans un délais de cent vingt jours suivant la promulgation de la Constitution, élaborera un code de défense du consommateur. $»^{15} \mathrm{C}^{\prime} e s t$ ainsi que fut adopté, le 11 septembre 1990, la loi 8 078, le Code de protection et de défense du consommateur (CDC). En harmonie avec ses fondations constitutionnelles, le Code «établit des normes de protection et de défense du consommateur, d'ordre publics et d'intérêt social. $»^{16}$

Ainsi, comme le souligne Claudia Lima Marques, l'État a le devoir, conformément à la Constitution, de s'assurer que tant les autorités judiciaire, législative qu'exécutive veillent, en prenant les dispositions appropriées, à la défense des intérêts des consommateurs. La protection du consommateur, droit fondamental au sein de l'ordre juridique brésilien, doit non seulement se matérialiser par une protection contre les actes de l'État mais aussi de façon positive et affirmative par l'État de manière à protéger le consommateur adéquatement dans ses relations privées. Bref, la protection du consommateur, en tant que droit fondamental, doit être respecté conformément aux normes infraconstitutionnelles (telles que le CDC) et aux exigences issues du concept de dignité humaine (tel que reconnu au sein de la Constitution). ${ }^{17}$

Ainsi, selon Mme. Lima Marques, l'inclusion de la protection du consommateur dans la Constitution de 1988 «est la garantie institutionnelle de l'existence et de l'effectivité du droit du consommateur au Brésil. $»^{18}$ De manière plus générale, l'inclusion du droit du consommateur dans la constitution brésilienne marquerait la reconstruction du droit privé brésilien selon des principes davantage sociaux créant ainsi un «droit privé solidaire. ${ }^{19}$ À cet égard, José Geraldo Brito Filomeno affirme que le système de protection du consommateur est bien plus qu'un ensemble de norme mais constitue un outil destiné à l'exercice de la citoyenneté. Ainsi, le CDC se veut être un microsystème juridique incluant des principes qui lui

\footnotetext{
13 Constituiçäo da República Federal do Brasil du 5 octobre de 1988, article 5, paragraphe XXXII

14 Constituição da República Federal do Brasil du 5 octobre de 1988, article 170, paragraphe V

15 Ato das Disposições Constitucionais Transitórias du 5 octobre de 1988, article 48.

16 Constituição da República Federal do Brasil du 5 octobre de 1988, article 1.

17 Antônio Herman Benjamin; Claudia Lima Marques, Leonardo Roscoe Bessa, Manual de Direito do Consumidor, São Paulo, Editora Revista dos Tribunais, 2008, p. 26.

18 Idem, p. 27

19 Ibid.
} 
sont propres (vulnérabilité du consommateur et destinataire final des produits et services), de nature interdisciplinaire (amalgamant droit constitutionnel, civil, pénal, administratif) et multidisciplinaire. ${ }^{20}$

\section{Principales dispositions du CDC}

Tout d'abord, au sein du second article, le consommateur est défini comme « toute personne physique ou juridique qui acquiert ou utilise un produit ou un service en tant que destinataire final. ${ }^{21}$ Quant à l'interprétation du concept de destinataire final, l'interprétation finaliste fut adoptée par le STJ (Tribunal Suprême de Justice), le DPDC (Département de protection et de défense du consommateur du Ministère de la Justice) et la Fondation Procon de São Paulo, entre autres institutions. Ainsi, la notion de consommateur est circonscrite «à celui qui acquiert (utilise) un produit pour son usage personnel et celui de sa famille; le consommateur ne serait pas un professionnel puisque, selon le CDC, doit être considéré titulaire un groupe de la société en position de vulnérabilité. ${ }^{22}$

Dans un deuxième temps, l'article 2 affirme que seront considérés comme consommateurs les individus ou la collectivité qui, même s'il est impossible de déterminer sa composition, sont intervenus dans une relation de consommation. ${ }^{23}$

Par ailleurs, notons que le CDC indique clairement, au sein de son article 4, les objectifs de la Politique nationale de relation de consommation soit, « de répondre aux besoins des consommateurs, de respecter leur dignité, leur santé et leur sécurité, de protéger leurs intérêts économiques, d'améliorer leur qualité de vie, ainsi que de rendre les relations de consommation transparentes et harmonieuses. ${ }^{24}$ Afin d'atteindre ces objectifs, le CDC établit une liste de principes de base : la reconnaissance de la vulnérabilité du consommateur (art. 4. I), la défense du consommateur par l'entremise des politiques étatiques (art. 4. II et VIII), la bonne foi objective et l'équilibre dans les relations entre les participants (art 4. III), l'information et l'éducation (art. 4. IV), le principe de qualité et de sécurité (art. 4. V et VII) et le principe de combat aux abus (art. 4 VI).

Toutefois, comme le souligne Mme. Lima Marques, après avoir reconnu constitutionnellement le consommateur et sa vulnérabilité, il était nécessaire pour l'État de définir une série de droits fondamentaux afin de' s'acquitter de son devoir de protection. ${ }^{25}$ Ainsi, le droit à la vie, à la santé et à la sécurité, sans aucun doute le plus important, est énoncé au premier paragraphe de l'article 6 . À cet égard, souligne

\footnotetext{
20 José Geraldo Brito Filomeno, Manual de Direitos do Consumidor, São Paulo, Editora Atlas S.A., 2005, p. 10.

21 Loi 8078 du 11 septembre de 1990, Código de Defesa do Consumidor, article 2.

2 Claudia Lima Marques; Antônio Herman Benjamin; Bruno Miragem, Comentários ao Código de Defesa do Consumidor, São Paulo, Editora Revista dos Tribunais, 2006, p. 84

23 Código de Defesa do Consumidor, article 2.

24 Código de Defesa do Consumidor, article 4

25 Benjamin, Marques, Bessa, p. 55
} 
Mme. Lima Marques, le CDC brise la séparation établie entre responsabilité contractuelle et extracontractuelle puisque tous les maillons de la chaine productive sont responsables de la sécurité du consommateur. ${ }^{26}$

Le paragraphe II de l'article 4 traite quant à lui du droit du consommateur à choisir librement. Afin de renforcer ce droit, plusieurs articles abordent les questions relatives aux relations précontractuelles et à la publicité et, conscient de l'agressivité des nouvelles techniques de commercialisation, mettent l'accent sur le droit à l'information et sur le droit à un délais de réflexion. ${ }^{27}$

Le droit à l'information figure comme troisième droit garanti par le CDC. Ainsi, en vertu du troisième paragraphe de l'article 6 , le consommateur doit pouvoir compter sur des informations claires et adéquates. Le Code spécifie que les informations quant à la quantité, à la composition, à la qualité, au prix et aux risques inhérents à la consommation du produit ou du service doivent êtres mentionnés. À cet égard, Mme. Lima Marques, souligne l'absolue nécessité de la norme afin d'équilibrer la relation de consommation entre le commerçant, expert en la matière, et le consommateur, en position de vulnérabilité. Elle ajoute que les dispositions incluses dans ce paragraphe régissent tant l'étape précontractuelle, que le moment de l'adhésion et les évènements post-contractuels. ${ }^{28}$ Pour leur part, les auteurs de l'avantprojet de loi 8078 (CDC) soulignent que ce paragraphe est destiné à détailler le paragraphe antérieur traitant de la liberté de choix du consommateur. ${ }^{29}$

Dans un quatrième temps, la «protection contre la publicité trompeuse et abusive, les méthodes commerciales coercitives et déloyales, tout comme contre les pratiques et les clauses abusives ou imposées sur l'offre de produits et de services» est assuré par l'article 6 du CDC. Développé plus en détail au sein des articles 30 à 38, mentionnons brièvement que tout message publicitaire se doit d'être véridique, de dévoiler les principaux attributs des produits et services en question, ne doit pas omettre d'attribut essentiel, d'être sobre et de respecter les normes d'étique. ${ }^{30}$ Pour sa part, Mme. Lima Marques défend que le quatrième paragraphe confirme le fait que la bonne foi, ici par l'entremise du caractère impératif de la transparence, constitue le principe fondateur du CDC. ${ }^{31}$ Notons aussi, que le principe de l'interprétation de la manière la plus favorable au consommateur est institué au sein du Code. ${ }^{32}$

Par la suite, le consommateur s'est vu attribuer le droit de modifier les clauses d'un contrat qui, même si elles ne sont pas abusives, établissent des prestations excessivement onéreuses ou qui, en raison d'un évènement extérieur, le serait devenu. En ce sens, la juge Nancy Andrighi a établi, dans une décision du STJ suite aux

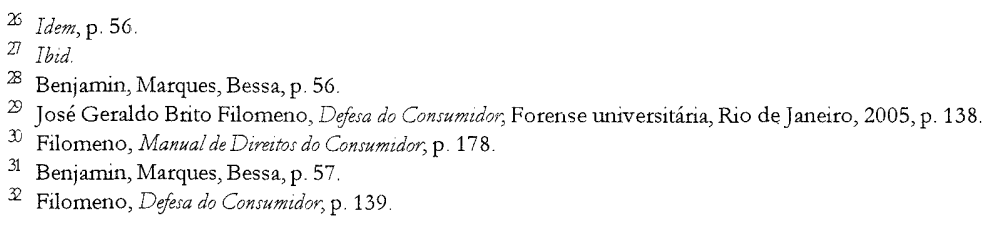


problèmes survenus suite à la dévaluation du Réal dans le cas de contrats libellés en dollars américains, que les consommateurs n'avaient pas à faire la preuve du caractère imprévisible de l'évènement puisque qu'il « suffit de démontrer de façon objective l'onérosité excessive du contrat pour le consommateur. $)^{33}$ De plus, contrairement à ce qui est explicité au sein du Code civil de 2002, le consommateur, en vertu du CDC, n'a pas à démontrer que le commerçant retire d'énormes avantages de ces clauses. Selon Mme. Lima Marques, ces dispositions peuvent êtres expliquées par le principe de bonne foi dans l'objectif que l'une des deux parties ne se voit ruinée. ${ }^{34} L$ 'importance de cet article est considérable dans un contexte où la législation brésilienne ne permet pas aux personnes physiques de recourir à la faillite. Ainsi, en vertu de cet article est mis de l'avant le devoir de renégociation des professionnels dans les cas de consommateurs surendettés. ${ }^{35}$

Par la suite, le sixième paragraphe de l'article 6 établit que les dommages patrimoniaux, moraux, individuels, collectifs et diffus devront êtres prévenus et réparés de façon effective. Le paragraphe suivant, quant à lui, garantit l'accès aux institutions judiciaires et administratives afin de prévenir et de réparer quelconque préjudice. En ce qui a trait à la prévention, soulignons l'importance du système de rappel, coordonné par le Département de protection et de défense du consommateur du Ministère de la justice à Brasília. ${ }^{36}$ Il est spécifié au sein de l'article 10 du CDC que les rappels doivent êtres effectués par l'entremise de la presse, de la radio et de la télévision, aux frais du fournisseur. Quant aux autorités étatiques, il est en leur devoir de superviser efficacement l'entrée de nouveaux produits sur le marché, au moyen des agences régulatrices par exemple (surveillance sanitaire, etc.). En ce qui a trait à la réparation des dommages subis, il est nécessaire que soit mis à la disposition du consommateur des recours modernes et efficaces. La possibilité que soient menés des recours collectifs est prévue dans la législation afin de faire respecter les intérêts « diffus» et « collectifs ${ }^{37}$

Au sein du septième paragraphe, le législateur veille à faciliter la défense des droits des consommateurs lorsque ceux-ci mettent de l'avant des allégations qui semblent véridiques ou sont «hyposufficients » en procédant à l'inversion du fardeau de la preuve. Ainsi, il est dans l'esprit du CDC de faciliter la défense du consommateur lorsqu'il est difficile pour le consommateur, considérant sa vulnérabilité, d'apporter une preuve en raison de sa haute technicité par exemple alors qu'il est plus facile pour le professionnel de démontrer le contraire. Ainsi, d'après Mme. Lima Marques, « exiger une preuve négative de la part du consommateur revient à l'obliger à payer deux fois pour le profit du professionnel dans le cadre d'activités comportant des

\footnotetext{
33 Recurso Especial 268.661/RJ, rapport de la juge Nancy Andrighi du 19/11/2001.

34 Benjamin, Marques, Bessa, p.59

35 Claudia Lima Marques, «Sugestões para una lei sobre o tratamento do superindividamento de pessoas físicas em contratos de crédito ao consumo \#. São Paulo, Revista de Direito do Consumidor no. 56 (octobre-décembre 2005), p. 22.

36 Leonardo Roscoe Bessa; Walter José Faiad de Moura, Manual de Direito do consumidor, Brasília, Ministério da Justiça, 2008, p. 61

37 Filomeno, Defesa do Consumidor, p. 141.
} 
risques : pour le prix payé tout comme pour les dommages subis. ${ }^{38}$ Dans un deuxième temps, le législateur prévoit que le consommateur hyposufficient, « terme emprunté par les auteurs du projet de loi à la doctrine du droit du travail qui considère le détenteur de la force de travail économiquement fragile face au détenteur du capital, son employeur, qui détient le pouvoir de subordination ${ }^{39}$, bénéficie aussi du renversement du fardeau de la preuve. Par ailleurs, l'article 2 de la loi 1060 de 5.2 .50 associe l'hypossufficience au « besoin » en affirmant que « doit se considérer en état de besoin, pour des fins légales, tous ceux dont la situation économique ne leur permet pas de payer les coûts du processus judiciaire et les honoraires de l'avocat, sans préjudice à sa capacité à se soutenir financièrement lui ou sa famille. $»^{40}$

Enfin, le dixième paragraphe garantit au consommateur le droit à obtenir de façon adéquate et efficace les services publics. Ainsi, il n'est fait aucune distinction quant à la nature des services essentiels en question, qu'ils soient « publics; ex-publics, privatisés [...]. . ${ }^{41}$ Ainsi, même dans le cas de services faisant l'objet d'une concession au secteur privé, les activités des ces entreprises sont toujours régies par le CDC lesquelles doivent se conformer à leur devoir de ne pas interrompre le service en question. ${ }^{42}$

\section{Système national de défense du consommateur}

Comme l'affirme M. Walder José Faiad de Moura, l'harmonisation des intérêts impliqués dans les relations de consommation, objectif de la Politique nationale des relations de consommation (PNRC), "réside en la matérialisation de la bonne foi dans sa triple émanation, de transparence, d'information et de confiance, afin, de manière diffuse et transversale, d'intégrer tous les agents du marché et le pouvoir étatique en un ensemble fonctionnant en synergie de façon à venir à bout de la marginalisation du consommateur. $\rangle^{43}$ De façon à favoriser cet objectif d'harmonisation et de favoriser l'application de la PNRC, fut adoptée le 20 mars 1997 le décret 2181 traitant de l'organisation du Système national de défense du consommateur (SNDC).

Sont membres du Système « le Secrétariat de droit économique du Ministère de la justice - SDE, par l'entremise de son Département de protection et de défense du consommateur - DPDC et toutes les autres entités fédérales, des états composant la fédération, du District Fédéral, municipales et civiles de défense du consommateur. ${ }^{44}$ Concrètement, le DPDC, les Procons, le Ministère public, l'aide judiciaire (Défensoria Pública), les autorités policières en matière de consommation (Delegacia de Defesa do Consumidor), les tribunaux des petites créances, les ONGs et les agences régulatrices font tous partie du SNDC.

\footnotetext{
33 Benjamin, Marques, Bessa, p. 62.

39 Filomeno, Defesa do Consumidor, p. 148

40 Idem, p. 149

41 Benjamin, Marques, Bessa, p. 62.

42 Idem, p. 63.

43 Walter José Faiad de Moura, «O Dever de Educar». São Paulo, Revista de direito do Consumidor, no. 60 (octobre-décembre 2006), p. 218

44 Décret 2182 du 20 mars de 1997 , article 2
} 


\section{Département de protection et de défense du consommateur}

Ainsi, suite au décret 2181, fut crée au sein du Ministère de la justice le DPDC afin de planifier, d'élaborer, de coordonner et d'exécuter la Politique nationale de protection et de défense du consommateur. ${ }^{45}$ Même s'il a comme mission de coordonner la politique nationale, il n'existe aucun lien hiérarchique entre le DPDC et les entités des états, en raison des articles 25 et 30 de la Constitution de 1988 garantissant l'autonomie de celles-ci dans leur champs de compétence. D'un autre côté, il incombe au DPDC de gérer le Système national d'informations sur la défense du consommateur (SINDEC). Sont compilées au sein du SINDEC les données quant au profil et aux réclamations des consommateurs. Bref, le SINDEC permet aux entités publiques de développer des politiques de défense du consommateur plus efficaces et tend à équilibrer les relations de consommation en mettant à la disposition des consommateurs des informations de première importance à propos des fouminisseurs contrevenant au CDC. ${ }^{46}$

\section{Procons}

Entités de défense du consommateur présentes au sein des états et de municipalités, les Procons ont comme objectif d'appliquer la législation consumériste, notamment par l'entremise de sanctions administratives. En plus des Procons liés à chacun des 26 états et du Procon actant dans le District Fédéral, il existe plus de 600 Procons municipaux (le Brésil compte plus de 5000 municipalités).

En plus d'appliquer les sanctions administratives suite à des activités de fiscalisation du marché, les Procons peuvent êtres appelés à participer à l'élaboration de mesures législatives, jouent un rôle clé pour l'éducation des consommateurs, par l'entremise de conférences et de publications, et mènent des recherches portant sur le marché de consommation. ${ }^{47}$

Les Procons sont aussi habilités à recevoir les plaintes des consommateurs qui, au lieu d'initier une action judiciaire, peuvent opter pour une séance de conciliation mené par le Procon. À cet égard, selon le modèle du Procon de São Paulo, le consommateur peut lui faire parvenir sa plainte par courrier, par fax ou en personne afin de lui permettre, après en avoir confirmé le bien-fondé, d'entrer en contact avec le commerçant ou le fournisseur de biens ou de services dans le but d'en venir à une entente. Dans le cas où le consommateur ne serait pas satisfait par la première intervention du Procon auprès du commerçant, une séance de conciliation est menée dans le but de parvenir à un compromis. Une fois une entente conclue, celle-ci a la valeur d'une décision judiciaire. Si toutefois aucun compromis n'est atteint, le consommateur devra se présenter devant le tribunal des petites créances afin de permettre à un juge de trancher de l'affaire. Ce processus

\footnotetext{
45 Décret 2 182, article 3, paragraphe 1.

46 Ministério da Justiça, Direito do Consumidor - SINDEC-Atresentagão, em ligne: http://www.mi.gov.br/dpdc/ (page consultée le 10 avril 2008).

47 Fundação PROCON de São Paulo, Objetizas, en ligne: http://www.procon.sp.gov.br/categoria asp?id=206/ (page consultée le 4 avril 2008).
} 
a pour qualité de favoriser l'accès à la justice et de contribuer à ce que ses droits soient effectivement respectés. D'un autre côté, afin d'obtenir des informations quant à leurs droits, les consommateurs peuvent entrer en contact avec le Procon par téléphone et par courriel. À titre d'exemple, le Procon de São Paulo a reçu au cours de l'année 2007 plus de 515000 demandes par l'entremise de tous les moyens mis à la disposition des consommateurs. En ce qui a trait aux interventions du Procon auprès des commerçants ou des fournisseurs suite aux plaintes de consommateurs, une entente fut atteinte dans 63\% des cas (14 359) tandis que $37 \%$ des plaintes n'ont mené à aucune entente (8472). Il est à noter que les services essentiels $(31,12 \%)$, les produits de consommation $(29,98 \%)$ et les questions financières $(25,07 \%)$ furent l'objet de la majorité des plaintes des consommateurs. ${ }^{48}$ Pour leurs parts, les 212 Procons municipaux de l'état de São Paulo furent consultés à plus de 750000 au cours de l'année 2007. La Fondation Procon de São Paulo emploie plus de 450 fonctionnaires tandis que l'on en compte près de 600 au sein des Procons municipaux de l'état de São Paulo. ${ }^{49}$

\section{Autres entités}

Quant au Ministère public, il a comme attribution de mener les actions judiciaires dans l'intérêt de la collectivité. Ainsi, constatant une atteinte à l'un des droits collectifs des consommateurs, le Ministère public peut intenter une action contre le commerçant fautif. Considérant que le Ministère public n'a pas comme attribution la défense des intérêts individuels des consommateurs, c'est à la Defensoria Pública (aide juridique), qu'il revient d'assurer la défense des individus dans le besoin. Dans la plupart des cas, les cas sont entendus devant les tribunaux des petites créances (juizados especiais civis). Enfin, advenant une infraction pénale, c'est à la Delegacia de Defesa do Consumidor que revient le devoir d'enquêter et d'acheminer le dossier au pouvoir judiciaire (Ministère public ou institutions judiciaires pertinentes). ${ }^{50}$

\section{Conclusion}

La promotion de la défense du consommateur fut constamment confrontée à une série de défis qui, heureusement, n'empêchèrent pas la création de solides fondations législatives et d'une panoplie d'organisations fort prometteuses. Si le $\mathrm{CDC}$ ne répond pas à tous les problèmes auxquels doivent faire face les consommateurs, pensons à l'absence de la faillite pour les personnes physiques, il reste néanmoins un outil sur lequel peut solidement se baser le Système national de protection du consommateur dans ses actions et son

\footnotetext{
48 Fundação PROCON de São Paulo, Cadastro de Reclamaçóes fundamentais 2007, São Paulo, Secretaria da Justiça e da Defesa da Cidadania, 2008, p. 2.

4. Entrevue avec Robson Santos Carlos, Département des relations institutionnelles de la Fundação PROCON de São Paulo, 8 avril 2008

50 Bessa et Moura, p. 23.
} 
développement futur. Quant aux institutions veillant à l'application et au développement du code, il ne fait pas de doute que le travail acharné des personnes les composant contribuera à fortifier encore davantage ces formidables outils et contribuera à faire gagner le Système national en harmonie et en efficacité. Bref, l'architecture avant-gardiste du système brésilien de défense du consommateur, notamment quant à son code et aux institutions le composant, doit légitimement figurer comme exemple à suivre pour la création ou la consolidation des autres systèmes nationaux. 